\title{
Glycosylation defects underlying fetal alcohol spectrum disorder: a novel pathogenetic model
}

\author{
"When the wine goes in, strange things come out" - S.T. Coleridge, The Piccolomini
}

\author{
M. Binkhorst • S. B. Wortmann • S. Funke • T. Kozicz • \\ R. A. Wevers • E. Morava
}

Received: 15 September 2011 /Revised: 7 November 2011 / Accepted: 14 November 2011 /Published online: 2 December 2011

(C) The Author(s) 2011. This article is published with open access at Springerlink.com

\begin{abstract}
Fetal alcohol spectrum disorder (FASD) is an umbrella term used to describe the craniofacial dysmorphic features, malformations, and disturbances in growth, neurodevelopment and behavior occurring in individuals prenatally exposed to alcohol. Fetal alcohol syndrome (FAS) represents the severe end of this spectrum. Many pathophysiological mechanisms have hitherto been proposed to account for the disrupted growth and morphogenesis seen in FAS. These include impaired cholesterol-modification of the Sonic hedgehog morphogen, retinoic acid deficiency, lipoperoxidative damage due to alcohol-induced reactive oxygen species combined with reduced antioxidant defences, and malfunctioning cell adhesion molecules. In this report, we propose a completely novel concept regarding the pathogenesis of FAS. Based on our observation that transferrin isoelectric focusing (TIEF) - the
\end{abstract}

Communicated by: Carlo Dionisi-Vici

Competing interest: None declared.

T. Kozicz, R. A. Wevers and E. Morava contributed equally.

S. B. Wortmann $\cdot$ R. A. Wevers $\cdot$ E. Morava $(\triangle)$

Institute for Genetic and Metabolic Disease (IGMD), Department

of Pediatrics, Radboud University Nijmegen Medical Centre,

Nijmegen, The Netherlands

e-mail: e.morava@cukz.umcn.nl

M. Binkhorst $\cdot$ S. B. Wortmann · E. Morava

Department of Pediatrics,

Radboud University Nijmegen Medical Centre,

Nijmegen, The Netherlands

R. A. Wevers

Laboratory of Genetic Endocrine and Metabolic Diseases at the

Department of Laboratory Medicine, Radboud University

Nijmegen Medical Centre,

Nijmegen, The Netherlands most widely used screening tool for congenital disorders of glycosylation (CDG) - was transiently abnormal in a newborn with FAS and a confirmed maternal history of gestational alcohol abuse, we came to believe that FAS exemplifies a congenital disorder of glycosylation secondary to alcohol-inflicted disruption of (N-linked) protein glycosylation. Various pieces of evidence were found in the literature to substantiate this hypothesis. This observation implies, among others, that one might need to consider the possibility of maternal alcohol consumption in newborns with transient glycosylation abnormalities. We also present an integrated pathophysiological model of FAS, which incorporates all existing theories mentioned above as well as our novel concept. This model highlights the pivotal role of disrupted isoprenoid metabolism in the origination of FAS.

\section{T. Kozicz}

Department of Cellular Animal Physiology at the Donders Centre of Neuroscience, Radboud University Nijmegen,

Nijmegen, The Netherlands

M. Binkhorst

Department of Pediatrics, Hieronymus Bosch Hospital,

's-Hertogenbosch, The Netherlands

S. Funke

Department of Neonatology, Obstetrics and Gynecology,

University of Pecs,

Pecs, Hungary 


$\begin{array}{ll}\text { Abbreviations } & \\ \alpha 1-A T & \text { Alpha-1-antitrypsin } \\ \text { CDG } & \text { Congenital disorders of glycosylation } \\ \text { CNS } & \text { Central nervous system } \\ \text { Dol-P } & \text { Dolichol phosphate } \\ \text { ER } & \text { Endoplasmic reticulum } \\ \text { FAS } & \text { Fetal alcohol syndrome } \\ \text { FASD } & \text { Fetal alcohol spectrum disorder } \\ \text { GA } & \text { Golgi apparatus } \\ \text { LLO } & \text { Lipid-linked oligosaccharide } \\ \text { PSA-NCAM } & \text { Polysialic acid neural cell adhesion molecules } \\ \text { RA } & \text { Retinoic acid } \\ \text { ROS } & \text { Reactive oxygen species } \\ \text { (T)IEF } & \text { (Transferrin) isoelectric focusing }\end{array}$

\section{Introduction}

In 1973, Jones and Smith launched the term fetal alcohol syndrome (FAS) to describe a characteristic pattern of altered fetal growth and morphogenesis due to maternal alcohol consumption during pregnancy (Jones and Smith 1973). Nowadays, the effects of prenatal alcohol exposure are collectively referred to as fetal alcohol spectrum disorder (FASD). The following anomalies are generally considered key elements of FASD: 1) prenatal and/or postnatal growth retardation, 2) dysmorphic facial features, mainly affecting the premaxillary region (smooth philtrum, thin vermillion border of the upper lip, short palpebral fissures), 3) alcoholrelated birth defects, such as cardiac, skeletal, renal and ocular malformations, and 4) alcohol-related neurodevelopmental disorders, which include structural central nervous system (CNS) abnormalities (microcephaly, partial or complete corpus callosum agenesis, cerebellar hypoplasia, and leptomeningeal heterotopias) as well as behavioral, cognitive, and motor disabilities (Van Balkom et al. 1996; Chudley et al. 2005). FAS epitomizes the most severe form of FASD. The degree to which offspring is affected by maternal alcohol ingestion depends on the timing and level of exposure, with more serious disruptions in fetus exposed to high concentrations of alcohol during the first weeks of pregnancy (Chudley et al. 2005; Guizzetti and Costa 2007; Kot-Leibovich and Fainsod 2009; Lipinski et al. 2010). FAS has an estimated incidence of 1-3 per 1000 live births and, as such, constitutes the principal cause of non-inherited mental retardation (Van Balkom et al. 1996; Chudley et al. 2005; Guizzetti and Costa 2007; Abdelrahman and Conn 2009). Multiple pathophysiological models have hitherto been proposed to account for the phenotypic appearance of FAS, most of which will be discussed below. None of these, however, fully clarifies the entire spectrum of alcoholrelated disorders.

\section{Observation and hypothesis}

In a series of 200 patients aged 0-24 months, prospectively screened for congenital disorders of glycosylation (CDG) because of hypotonia and/or psychomotor developmental delay, we encountered a male newborn demonstrating a false positive screening result. This boy was the first child of healthy parents. There was a positive history of smoking and alcohol consumption during pregnancy. He was born at term, small for gestational age and treated for perinatal group B streptococcal disease. He had abnormal alpha-1antitrypsin ( $\alpha 1-\mathrm{AT})$ electrophoresis, neonatal hypoglycemia, elevated liver transaminases and developed axial hypotonia, increased deep tendon reflexes and mild peripheral spasticity. Initial screening at the age of 1 week showed abnormal transferrin isoelectric focusing (TIEF). At the age of 3 months, biochemical re-evaluation showed normal results for TIEF, isoelectric focusing of thyroglobulin and $\alpha 1-\mathrm{AT}$, as well as normal phosphomannose-mutase (PMM2) and phosphomannose-isomerase (PMI) enzyme analyses. Based on characteristic dysmorphic features (short palpebral fissures, epicanthal folds, hypoplastic midface, short nose, long philtrum, inverted nipples, short terminal phalanges of the fifth fingers and atrial septal defect) and the pregnancy history, he was later diagnosed with FAS. His clinical course was further complicated by developmental delay, speech delay and epilepsy.

The false positive screening for $\mathrm{CDG}-$ i.e., the transiently abnormal TIEF pattern - in this patient with a later diagnosis of FAS made us aware of a possible pathophysiological link between CDG and FAS. We therefore studied the literature to find evidence to verify our hypothesis that FAS can be regarded as a CDG secondary to prenatal alcohol exposure. Moreover, we composed a comprehensive pathophysiological model of FAS, which incorporates all existing theories as well as our own novel concept.

\section{Existing theories}

Cholesterol deficiency and Sonic hedgehog dysfunction

Cholesterol is an end product of the mevalonate pathway (Edison and Muenke 2004). For later discussion, it is important to mention that intermediates of this pathway are commonly referred to as isoprenoids, for they contain a variable number of isoprene units. Smith-Lemli-Opitz syndrome (SLOS) is the most common inherited defect in this biosynthetic pathway. It is caused by mutations in the 7dehydrocholesterol reductase gene, the enzyme product of which catalyzes the final reaction of cholesterol biosynthesis, i.e., the conversion of 7-dehydrocholesterol to cholesterol. SLOS and FAS have strikingly similar phenotypes, 
both in humans and animal models, which corroborates the contention that cholesterol deficiency plays a pathophysiological role in FAS (Guizzetti and Costa 2007). Cholesterol is crucial for normal development. In addition to being a regulator of membrane fluidity and permeability, a precursor for steroid hormones, a substrate for glial cell proliferation and synaptogenesis, and a constituent of myelin sheaths and cholesterol-rich plasma rafts involved in signal transduction, covalent binding of cholesterol to Hedgehog morphogens is a posttranslational modification required for proper activity of these latter proteins (Edison and Muenke 2004; Guizzetti and Costa 2007). Sonic hedgehog (Shh) guides cell proliferation, differentiation and morphogenesis of the CNS, craniofacial region, musculoskeletal structures and viscera during embryonic and fetal development. Reduced cellular availability of cholesterol results in a drastic decline in Shh signal transduction in mice (Edison and Muenke 2004). Ethanol has been shown to inhibit the mevalonate pathway, and thus cholesterol synthesis (Cottalasso et al. 1998; Li et al. 2007). Furthermore, chicken and mouse embryos exposed to ethanol displayed reduced Shh signaling (Guizzetti and Costa 2007). These findings led to the belief that alcoholdependent inhibition of the cholesterol-modification of Shh produces the morphologic defects seen in FAS. To study the interrelationship among prenatal alcohol exposure, cholesterol depletion, impairment of Shh signaling and FAS, investigators commonly use animal models of holoprosencephaly (HPE). HPE is not only the classic brain defect seen in families with mutations in Shh, but also a manifestation in severe cases of SLOS and FAS, and can be experimentally provoked by treating animal embryos with ethanol, inhibitors of cholesterol biosynthesis, and inhibitors of Shh signal transduction (Edison and Muenke 2004; Li et al. 2007; Lipinski et al. 2010). Li et al. have provided the most conclusive evidence substantiating the link between cholesterol deficiency and FAS (Li et al. 2007). They demonstrated that alcohol treatment of zebrafish embryos caused a dose-dependent reduction in cholesterol content, decreased cholesterol-modification of Shh and a loss of Shh signal transduction, resulting in a FAS-like phenotype (i.e., HPE, cyclopia, and craniofacial hypoplasia). These alcoholinduced effects could be rescued by supplementing the zebrafish embryos with cholesterol.

Retinol deficiency and impaired retinoic acid formation

Retinoic acid (RA) is another eminent morphogen, governing cell differentiation and early embryonic patterning. RA is, among others, responsible for proper development of the craniofacial region and skeleton in vertebrates. In experimental settings, both RA deficiency - either due to a vitamin A-deficient diet or mutations in RA-synthesizing enzymes and RA-receptor defects produce phenotypes strongly resembling FAS (Deltour et al. 1996; Kot-Leibovich and Fainsod 2009). Ethanol has been linked to RA depletion as follows. Retinol - an isoprenoid - is converted to RA in a two-step oxidative reaction. First, retinol is oxidized by alcohol dehydrogenase $(\mathrm{ADH})$, producing the intermediate retinaldehyde. Second, retinaldehyde dehydrogenase (RALDH) catalyzes the conversion of retinaldehyde to RA. Ethanol detoxification involves identical oxidation steps. Ethanol is initially oxidized to acetaldehyde through the action of $\mathrm{ADH}$, followed by formation of acetic acid using ALDH as a catalyst. Evidence is available showing that ethanol competitively inhibits both ADH and RALDH, resulting in impaired RA biosynthesis, which leads to developmental malformations mimicking FAS (Deltour et al. 1996; Kot-Leibovich and Fainsod 2009). Lack of retinol substrate, e.g., due to lipid peroxidation, as will be discussed in the following paragraph, may further thwart RA biosynthesis.

\section{Damage due to reactive oxygen species}

Prolonged exposure to ethanol induces the microsomal ethanol oxidizing system (cytochrome P450, isoenzyme CYP2E1), which generates reactive oxygen species (ROS) (Cottalasso et al. 1998). Although mRNA for P450 2E1 could neither be detected in murine embryos at the time of gastrulation and neurulation - the developmental stages during which ethanol exposure causes cerebral and craniofacial malformations - nor in human embryonic hepatic tissue during the first half of gestation (Deltour et al. 1996), this does not exclude microsomal ROS production at other sites or different developmental stages. Both cholesterol and dolichol are isoprene derivates, and, as such, are particularly vulnerable to the lipoperoxidative effects of ethanol-induced ROS. In addition to the consequences of cholesterol and dolichol depletion outlined above and below, respectively, degradation of these vital membrane constituents affects plasma membrane integrity and vesicular trafficking (Cottalasso et al. 1998; Guizzetti and Costa 2007). Retinol, and its precursor $\beta$-carotene, also consisting of isoprene motifs, can equally suffer from ROS attack, especially in the absence of antioxidants such as vitamin $\mathrm{E}$, vitamin $\mathrm{C}$ and glutathione peroxidase. The generally poor nutritional state of alcoholic mothers usually entails hypovitaminosis. Also, ethanol has been shown to decrease glutathione peroxidase activity (Cohen-Kerem and Koren 2003). Hence, ethanol intake does not only cause oxidative stress, it also curtails antioxidant defences. The contribution of ROS-induced damage to ethanol teratogenesis is substantiated by evidence from several animal studies showing that supplementation of antioxidants (e.g., vitamin E, flavonoids, folic acid, and $\beta$-carotene) attenuates oxidative stress and, thereby, diminishes fetotoxicity (Cohen-Kerem and Koren 2003; Wentzel et al. 2006). 
Interference with neural cell adhesion molecules

During embryonic CNS development, neural cell adhesion molecules (NCAMs) guide processes as neuron-glia interaction, synaptogenesis, neuronal migration, growth and morphogenesis (Miñana et al. 2000). In utero, NCAMs require posttranslational glycosylation - i.e., the addition of multiple polysialic acid (PSA) residues, mediated by sialyltransferase (ST) enzymes in the Golgi apparatus (GA) - in order to be properly transferred to the plasma membrane and fulfill their function in enabling neural cell migration. Prenatal alcohol exposure inhibits ST activity and leads to cytoplasmic retention of PSA-NCAMs. The resultant reduction in cell surface expression and functionality of PSA-NCAMs is thought to underlie the migrational errors, heterotopias and other morphological brain defects seen in FAS (Miñana et al. 2000). Another important cell adhesion molecule, regulating cell-cell and cell-matrix interactions and signaling in the developing brain, is L1CAM, which belongs to the immunoglobin superfamily. L1CAM contains 21 sites for $\mathrm{N}$-linked glycosylation, a feature that will acquire significance in the following paragraph. Patients with mutations in L1 display a phenotype known as CRASH syndrome (Corpus callosum hypoplasia/agenesis, psychomotor Retardation, Adducted thumbs, Spastic paraparesis, Hydrocephalus; MIM *308840). Additional neuropathologic features observed in these patients include hypoplasia of the cerebellar vermis and ocular anomalies, such as nystagmus, ptosis, optic nerve hypoplasia and retinal vessel tortuosity. This phenotype is rather reminiscent of FAS. The supposition that FAS results from ethanol-induced aberrations in L1 function has gained plausibility by the observation that ethanol indeed disrupts L1-mediated cell adhesion and neurite outgrowth in various in vitro studies (Bearer 2001).

\section{A novel concept}

Dolichol deficiency and disruption of protein glycosylation

We are, to our knowledge, the first authors to comprehensively reflect on the concept of FAS exemplifying a congenital disorder of glycosylation (CDG) secondary to prenatal alcohol exposure. To better understand our theory, we need to discuss the processes involved in protein glycosylation (Mohamed et al. 2011a; Achouitar et al. 2011). In N-linked glycosylation, a branched oligosaccharide chain is cotranslationally assembled on a lipid anchor, i.e., dolichol phosphate (Dol-P), attached to the membrane of the endoplasmic reticulum (ER). The resultant complex is known as lipidlinked oligosaccharide (LLO). Next, the preassembled oligosaccharide is transferred en bloc to asparagine residues at glycosylation consensus sites of the nascent protein. The glycoprotein is subsequently translocated to the GA by vesicular transport, where several enzymes - of which sialyltransferase (ST), galactosyltransferase (GT), and sialidase are worthwhile mentioning in the light of the present discussion - modify the oligosaccharide structure. The final protein-bound oligosaccharide is conventionally depicted as a biantennary structure with sialic acid residues at the end of both antennae. Inherited defects in N-linked glycosylation are divided in two subtypes (Morava et al. 2008; Mohamed et al. 2011b; Achouitar et al. 2011). Type I CDG involves defects in LLO assembly and oligosaccharide transfer to the nascent protein, resulting in proteins lacking complete oligosaccharides (Mohamed et al. 2011a; Achouitar et al. 2011). Type II CDG encompasses disturbances in the GAlocated processing of the protein-bound oligosaccharide, usually giving rise to oligosaccharides with truncated antennas. Screening for CDG is performed with isoelectric focusing of the serum glycoprotein transferrin (TIEF) (Morava et al. 2008; Mohamed et al. 2011b; Achouitar et al. 2011). In healthy individuals, transferrin predominantly occurs as tetrasialotransferrin, for it has two biantennary oligosaccharide side chains with a total of four terminal sialic acid residues. A TIEF type I pattern shows decreased tetrasialotransferrin and increased di- and asialotransferrin, whereas a TIEF type II pattern additionally features tri- and monosialotransferrin.

Cottalasso et al. demonstrated that ethanol treatment causes a profound reduction in the dolichol (phosphate) content of rat liver microsomes and GA (Cottalasso et al. 1998). They also showed that the mechanism underlying this decrease involves both inhibition of dolichol biosynthesis (i.e., the mevalonate pathway) and dolichol peroxidation due to ROS. A sufficient level of microsomal Dol-P is a prerequisite for the initiation of N-linked glycosylation. Thus, ethanol exposure may seriously impede glycoprotein synthesis and secretion during fetal life. Ethanol also affects mannosyltransferase (MT) activity, an enzyme mediating mannosylation of Dol-P during the build-up of the LLO (Cottalasso et al. 1998). Furthermore, ST and GT are inhibited, while sialidase activity is stimulated in response to ethanol administration (Cottalasso et al. 1998; Flahaut et al. 2003). These latter effects of ethanol, causing disruption of terminal sialylation during the second phase of N-linked glycosylation in the GA, are probably of minor importance, considering that carbohydrate-deficient transferrin (CDT) the best indicator of chronic alcohol abuse currently available - mainly consists of di- and asialotransferrin (type I TIEF pattern), indicating defects in LLO assembly during the first phase of N-linked glycosylation in the ER (Landberg et al. 1995; Flahaut et al. 2003). We recently witnessed a transiently abnormal (type I) IEF pattern of serum transferrin and $\alpha 1-\mathrm{AT}$ in a male newborn at the age of one week, screened for CDG because of hypotonia, 
dysmorphic features, hypoglycemia, elevated liver enzymes, and spasticity. At the age of three months, IEF patterns were normal. Following confirmation of maternal alcohol abuse during pregnancy, FAS was diagnosed. We speculate that the anomalous IEF patterns observed shortly after birth were due to ethanol-induced glycosylation defects in utero.

Tomás et al. reported that the glycosylation machinery is indeed targeted by ethanol in rat astrocytes, thereby putatively disrupting brain development. Deleterious effects of ethanol on the glycosylation process have also been demonstrated in hepatocytes and neurons (Tomás et al. 2002). The link between ethanol-induced glycosylation defects and the (neuro)developmental perturbations seen in FAS is easily understood when one realizes that many proteins involved in cell recognition, adhesion, migration and signaling are in fact glycoproteins. Among these are L1, PSA-NCAM, Notch, $\alpha$ dystroglycan, and various growth factor receptors (Tomás et al. 2002; Edison and Muenke 2004; Achouitar et al. 2011).

There are many phenotypic similarities between FAS and CDG. Both are generally characterized by growth retardation, developmental delay, intellectual disability, ataxia, hypotonia, seizures, and dysmorphic facial features (e.g., hypertelorism, short upturned nose, malformed ears, and midface hypoplasia). Neuropathologic resemblances include microcephaly, cerebral atrophy, cerebellar (vermis) hypoplasia, corpus callosum hypoplasia/agenesis, and migration errors (Jones and Smith 1973; Van Balkom et al. 1996; Chudley et al. 2005; Morava et al. 2009; Achouitar et al. 2011). Of special interest are the ocular anomalies common to both entities. Coloboma, microphthalmia (causing short

Fig. 1 Integrated pathophysiological model of fetal alcohol syndrome (FAS). ADH, alcohol dehydrogenase; RALDH, retinaldehyde dehydrogenase; LLO, lipid-linked oligosaccharide; CNS, central nervous system; PSA-NCAM, polysialic acid neural cell adhesion molecules; L1CAM, L1 cell adhesion molecule

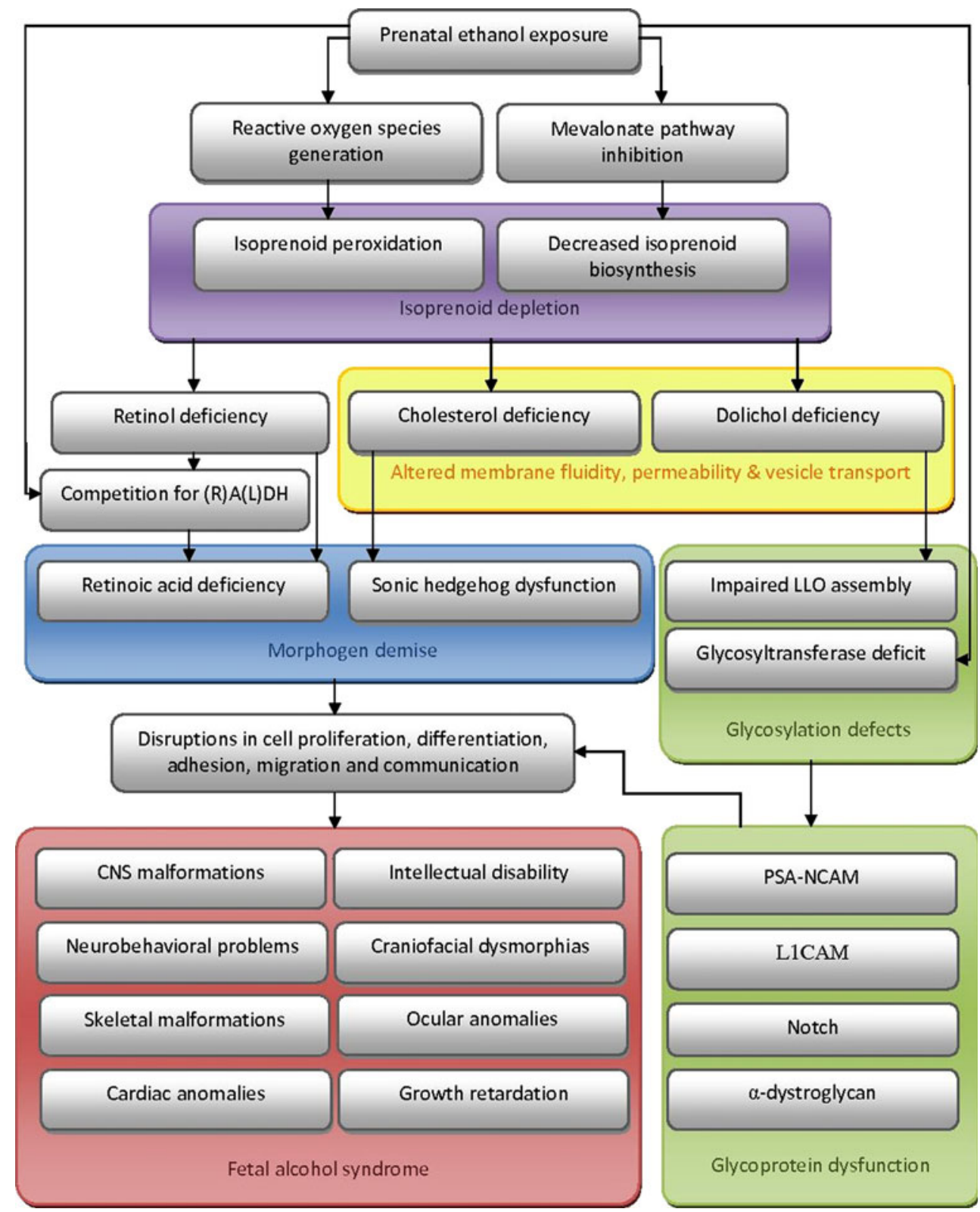


palpebral fissures), optic nerve hypoplasia, nystagmus, and strabismus (esotropia) are frequently observed in patients with FAS (Abdelrahman and Conn 2009). Uveal coloboma and microphthalmia serve as measures of effect in murine models of ethanol teratogenicity (Parnell et al. 2006). All these eye abnormalities are also encountered in CDG patients (Morava et al. 2010; Achouitar et al. 2011; Mohamed et al. 2011a). We have to note that certain nonstructural abnormalities specific to CDG, e.g., coagulopathy and endocrinopathy, are not present in FAS, because active disruption of glycosylation ceases at birth in the latter, after which proper synthesis of coagulatory and hormonal glycoproteins presumably resumes.

\section{A unifying theory and future directions}

We propose an integrated pathophysiological model for the development of FAS (Fig. 1), incorporating all previously mentioned mechanisms. We emphasize the central role of ethanol-induced disruption of isoprenoid metabolism. Deficient biosynthesis and increased degradation of important isoprene derivatives (cholesterol, dolichol, and retinol) contribute to the pathogenesis of FAS.

Although various components of our model have already been tested, as described above, further studies could corroborate the pivotal role of perturbed isoprenoid metabolism in FAS. HMG-CoA reductase inhibitors (statins) and nitrogen-containing bisphosphonates inhibit crucial enzymes in the mevalonate pathway, thereby downregulating the biosynthesis of dolichol and cholesterol. Lipophilic statins have been shown to reach the embryo when ingested by pregnant women (Edison and Muenke 2004). Offspring of animals gestationally exposed to (high doses of) these drugs can be monitored for the development of a phenotype mimicking FAS. It should be noted that rodents are not suitable for these experiments, since these animals are not susceptible to the cholesterol-lowering effects of statins (Edison and Muenke 2004). In vitro and in vivo studies are needed, in which cholesterol, dolichol, and/or $\beta$-carotene/retinol are administered simultaneously with ethanol in order to assess whether these isoprenoid substances ameliorate ethanolinflicted abnormalities. IEF can be used to detect abnormal glycosylation of several glycoproteins (e.g., transferrin, $\alpha 1$ AT, and thyroglobulin) in the (cord) blood of newborns with characteristic FAS features and a history of maternal gestational alcohol abuse. Inasmuch as the ethanol-induced CNS and craniofacial malformations are thought to originate during gastrulation and neurulation, it may be valuable to determine the microsomal Dol-P level, MT activity, and glycosylation status of several glycoproteins (PSA-NCAM, L1, growth factor receptors) in early embryonic neuronal tissue of an animal model of FAS. Furthermore, when alcohol 'goes in' pregnant women and animals, various antioxidants may be used to prevent 'strange things coming out' of them. Finally, we suggest to consider the possibility of maternal alcohol abuse during pregnancy in newborns with transient glycosylation abnormalities.

Open Access This article is distributed under the terms of the Creative Commons Attribution Noncommercial License which permits any noncommercial use, distribution, and reproduction in any medium, provided the original author(s) and source are credited.

\section{References}

Abdelrahman A, Conn R (2009) Eye abnormalities in fetal alcohol syndrome. Ulster Med J 78:164-165

Achouitar S, Mohamed M, Gardeitchik T et al. (2011) Nijmegen paediatric CDG rating scale: a novel tool to assess disease progression. J Inherit Metab Dis 34:923-927

Bearer CF (2001) L1 Cell adhesion molecule signal cascades: targets for ethanol developmental neurotoxicity. Neurotoxicol 22:625-633

Chudley AE, Conry J, Cook JL, Loock C, Rosales T, LeBlanc N (2005) Fetal alcohol spectrum disorder: Canadian guidelines for diagnosis. CMAJ 172:S1-S21

Cohen-Kerem R, Koren G (2003) Antioxidants and fetal protection against ethanol teratogenicity I. Review of the experimental data and implications to humans. Neurotoxicol Teratol 25:1-9

Cottalasso D, Bellocchio A, Pronzato MA et al. (1998) Effect of ethanol administration on the level of dolichol in rat liver microsomes and Golgi apparatus. Alcohol Clin Exp Res 22:730-737

Deltour L, Ang HL, Duester G (1996) Ethanol inhibition of retinoic acid synthesis as a potential mechanism for fetal alcohol syndrome. FASEB J 10:1050-1057

Edison RJ, Muenke M (2004) Mechanistic and epidemiologic considerations in the evaluation of adverse birth outcomes following gestational exposure to statins. Am J Med Genet 131A:287-298

Flahaut C, Michalski JC, Danel T, Humbert MH, Klein A (2003) The effects of ethanol on the glycosylation of human transferrin. Glycobiology 13:191-198

Guizzetti M, Costa LG (2007) Cholesterol homeostasis in the developing brain: a possible new target for ethanol. Hum Exp Toxicol 26:355-360

Jones KL, Smith DW (1973) Recognition of the fetal alcohol syndrome in early infancy. Lancet 2:999-1001

Kot-Leibovich H, Fainsod A (2009) Ethanol induces embryonic malformations by competing for retinaldehyde dehydrogenase activity during vertebrate gastrulation. Dis Model Mech 2:295-305

Landberg E, Påhlsson P, Lundblad A, Arnetorp A, Jeppsson JO (1995) Carbohydrate composition of serum transferrin isoforms from patients with high alcohol consumption. Biochem Biophys Res Commun 210:267-274

Li YX, Yang HT, Zdanowicz M et al. (2007) Fetal alcohol exposure impairs hedgehog cholesterol modification and signaling. Lab Invest 87:231-240

Lipinski RJ, Godin EA, O’Leary-Moore SK, Parnell SE, Sulik KK (2010) Genesis of teratogen-induced holoprosencephaly in mice. Am J Med Genet C Semin Med Genet 154C:29-42

Miñana R, Climent E, Barettino D, Segui JM, Renau-Piqueras J, Guerri C (2000) Alcohol exposure alters the expression pattern of neural cell adhesion molecules during brain development. J Neurochem 75:954-964 
Mohamed M, Guillard M, Wortmann SB et al. (2011a) Clinical and diagnostic approach in unsolved CDG patients with a type 2 transferrin pattern. Biochim Biophys Acta 1812:691698

Mohamed M, Cantagrel V, Al-Gazali L, Wevers RA, Lefeber DJ, Morava E (2011b) Normal glycosylation screening does not rule out SRD5A3-CDG. Eur J Hum Genet 19. doi:10.1038/ ejhg. 2010.260

Morava E, Lefeber DJ, Urban Z et al. (2008) Defining the phenotype in an autosomal recessive cutis laxa syndrome with a combined congenital defect of glycosylation. Eur J Hum Genet 16:28-35

Morava E, Wevers RA, Willemsen MA, Lefeber D (2009) Cobblestone-like brain dysgenesis and altered glycosylation in congenital cutis laxa, Debré type. Neurology 73:1164
Morava E, Wevers RA, Cantagrel Vet al. (2010) A novel cerebello-ocular syndrome with abnormal glycosylation due to abnormalities in dolichol metabolism. Brain 133:3210-3220

Parnell SE, Dehart DB, Wills TA et al. (2006) Maternal oral intake mouse model for fetal alcohol spectrum disorders: ocular defects as a measure of effect. Alcohol Clin Exp Res 30:1791-1798

Tomás M, Fornas E, Megías L et al. (2002) Ethanol impairs monosaccharide uptake and glycosylation in cultured rat astrocytes. J Neurochem 83:601-612

Van Balkom ID, Gunning WB, Hennekam RC (1996) Fetal alcohol syndrome: an unrecognized cause of intellectual handicap and problem behavior in the Netherlands. Ned Tijdschr Geneeskd 140:592-595

Wentzel P, Rydberg U, Eriksson UJ (2006) Antioxidative treatment diminishes ethanol-induced congenital malformations in the rat. Alcohol Clin Exp Res 30:1752-1760 